\title{
RATIONAL SURFACES WITH INFINITE AUTOMORPHISM GROUP AND NO ANTIPLURICANONICAL CURVE
}

\author{
BRIAN HARBOURNE
}

\begin{abstract}
Counterexamples are given to a conjecture communicated to me by I. Dolgachev and E. Looijenga of M. Gizatullin that every rational surface with an infinite automorphism group should have an antipluricanonical curve.
\end{abstract}

There are many cases of complete smooth rational surfaces (over an algebraically closed field) which have infinitely many automorphisms. Examples include all rational relatively minimal models, certain Coble surfaces [D1] and certain blowings-up of $P^{2}$ having an irreducible and reduced anticanonical curve [H1]. These examples all share the property that a negative multiple of the canonical class contains an effective divisor (i.e., an antipluricanonical curve). This suggests that perhaps any rational surface with an infinite automorphism group would have an antipluricanonical curve. We give two counterexamples to show that this need not be so.

There are two quite different ways in which the automorphism group $\operatorname{Aut}(X)$ of a complete, smooth, rational surface $X$ can be infinite. Of course, Aut $(X)$ acts on the Picard group Pic $(X)$ of $X$. The image $\operatorname{Aut}^{*}(X)$ and the kernel Aut ${ }^{\#}(X)$ of the representation of $\operatorname{Aut}(X)$ on $\operatorname{Pic}(X)$ give an exact sequence:

$$
1 \rightarrow \operatorname{Aut}^{\#}(X) \rightarrow \operatorname{Aut}(X) \rightarrow \operatorname{Aut}^{*}(X) \rightarrow 1 .
$$

M. Gizatullin has conjectured that the occurrence of an antipluricanonical curve is in some way characteristic of those surfaces $X$ for which $\operatorname{Aut}^{*}(X)$ is infinite. We give a counterexample in which $\operatorname{Aut}^{*}(X)$ is infinite and yet $X$ has no antipluricanonical curve. However, the answer is not known to the strong form of the conjecture, posed now as the following question: If $\operatorname{Aut}^{*}(X)$ is infinite but $X$ has no antipluricanonical curve, is there then a birational morphism $\varphi$ of $X$ to a surface $Y$ having an antipluricanonical curve and an infinite subgroup $G \subset \operatorname{Aut}^{*}(Y)$, such that $G$ lifts via $\varphi$ to $X$ ?

Besides giving the counterexample mentioned above we show for any smooth rational surface $X$ that the groups $\operatorname{Aut}^{\#}(X)$ and $\operatorname{Aut}^{*}(X)$ are never both infinite, and for completeness we also give an example of a surface $X$ having no antipluricanonical curve but for which Aut ${ }^{\#}(X)$ is infinite. Throughout this paper $X$ refers to a complete, smooth, rational surface over an algebraically closed field $k$ of arbitrary characteristic.

1. $\operatorname{Aut}^{\#}(X)$ and $\operatorname{Aut}^{*}(X)$ are never both infinite. We begin with the following lemma, in which by an exceptional curve on $X$ we mean a one-dimensional fibre of a birational morphism of $X$ to a smooth surface.

Received by the editors October 21, 1985 and, in revised form, February 5, 1986.

1980 Mathematics Subject Classification (1985 Revision). Primary 14M20; Secondary $14 J 25$. 
Lemma (1.1). If $\operatorname{Aut}^{*}(X)$ is infinite, then $X$ must contain infinitely many exceptional curves.

ProOF. Let $G(X)$ be the subgroup of $\operatorname{Aut}(X)$ of all automorphisms whose induced action on the set of classes in $\operatorname{Pic}(X)$ of exceptional curves is trivial. Then $G(X)$ descends to a group of automorphisms of any relatively minimal model $X^{\prime}$ of $X$ (i.e., any birational morphism $X \rightarrow X^{\prime}$ is $G(X)$-equivariant). Denoting $G(X) / \operatorname{Aut}^{\#}(X)$ by $G^{*}(X), G^{*}(X)$ is isomorphic to $G(X) /\left(\operatorname{Aut}^{\#}\left(X^{\prime}\right) \cap G(X)\right)$ which is isomorphic to a subgroup of $G^{*}\left(X^{\prime}\right) \cong \operatorname{Aut}^{*}\left(X^{\prime}\right)$ which itself is $\mathbb{Z} / 2 \mathbb{Z}$ if $X^{\prime}=\mathbf{P}^{1} \times \mathbb{P}^{1}$ and is trivial otherwise. Since $\operatorname{Aut}^{*}(X)$ is infinite, we see $X \neq X^{\prime}$, and, since any blowing-up of $P^{1} \times P^{1}$ can be blown down to $\mathrm{P}^{2}$, we may assume $X^{\prime} \neq \mathbb{P}^{1} \times \mathbb{P}^{1}$; i.e., we may assume that $G^{*}\left(X^{\prime}\right)$, and thus $G^{*}(X)$, is trivial. Again, $\operatorname{Aut}^{*}(X) \cong \operatorname{Aut}^{*}(X) / G^{*}(X)$ is infinite, which, being a group of permutations of the classes of exceptional curves, implies that there are infinitely many exceptional curves.

COROLlaRY (1.2). If the set of exceptional curves on $X$ is infinite (for example, if $\operatorname{Aut}^{*}(X)$ is infinite), then there is a birational morphism $X \rightarrow \mathrm{P}^{2}$; i.e., $\mathrm{P}^{2}$ is a relatively minimal model of $X$.

Proof. We note by the lemma that if $\operatorname{Aut}^{*}(X)$ is infinite, then $X$ has infinitely many exceptional curves. By perhaps contracting some of these exceptional curves we obtain a birational morphism to a surface $Y$ having infinitely many irreducible exceptional curves. But it follows from Theorem 5 of $[\mathbf{N}]$ that $\mathbf{P}^{2}$ is a relatively minimal model of $Y$, and hence also of $X$.

The next result is the key step in showing that $\operatorname{Aut}^{*}(X)$ and $\operatorname{Aut}^{\#}(X)$ are never both infinite.

Proposition (1.3). If $\operatorname{Aut}^{\#}(X)$ is infinite, then $X$ has only finitely many exceptional curves.

Proof. Suppose that $\operatorname{Aut}^{\#}(X)$ is infinite but that $X$ has infinitely many exceptional curves. Then, as in the proof of Corollary (1.2), by contracting some exceptional curves if necessary we obtain a birational morphism $X \rightarrow Y$ such that $Y$ has infinitely many irreducible exceptional curves. Moreover, the elements of $\operatorname{Aut}^{\#}(X)$ all descend to automorphisms of $Y$. In particular, $\operatorname{Aut}^{\#}(Y)$ is still infinite. We also note that Corollary (1.2) implies that there is a birational morphism $\psi: Y \rightarrow \mathbb{P}^{2}$.

Since $Y$ has infinitely many irreducible exceptional curves, the set of their images under $\psi$ must have elements of arbitrarily large degree. Consider an irreducible exceptional curve $E$ on $Y$ for which $\psi(E)$, which we denote $E^{\prime}$, has degree at least 2 . Since Aut ${ }^{\#}(Y)$ takes each exceptional curve on $Y$ to itself, there is a homomorphism $\operatorname{Aut}^{\#}(Y) \rightarrow \operatorname{Aut}(E)$. This restriction homomorphism is an injection. For say an element $\alpha^{\prime}$ of the former restricts to the trivial automorphism of $E$. But $\psi$ is Aut $^{\#}(Y)$-equivariant, so $\alpha^{\prime}$ descends to an automorphism $\alpha$ of $\mathbf{P}^{2}$ which fixes each point of $E^{\prime}$. Pairs of points of $E^{\prime}$ determine lines, so $\alpha$ takes each line to itself. But each point of any given line lies on another line and is therefore a fixed point of $\alpha$, so $\alpha$ acts trivially on $\mathrm{P}^{2}$, and hence $\alpha^{\prime}$ must be the identity of $\operatorname{Aut}^{\#}(Y)$.

Consider the identity component $\operatorname{Aut}^{0}(Y)$ of $\operatorname{Aut}^{\#}(Y)$. If it is not finite, then it is a positive dimensional algebraic group and so contains an irreducible algebraic 
subgroup $G$ of dimension one. This is because if $\operatorname{Aut}^{0}(Y)$ has positive rank then it contains a torus, while otherwise it is unipotent and thus has a nilpotent subgroup of dimension one $[\mathbf{H u}$, pp. 122, 137]. But the preceding paragraph showed that $G$ maps injectively to $\operatorname{Aut}(E)$ for any irreducible exceptional curve $E$ having $\psi(E)$ of degree at least 2, and therefore any such curve $E$ is the closure of an orbit of $G$ acting on $Y$. Since there are infinitely many such curves $E$, there are infinitely many $G$-orbits whose closures are irreducible exceptional curves.

The main result of $[\mathbf{R}]$ now shows that there is a $G$-invariant dense open subvariety $V$ of $Y$ for which the quotient of $Y$ by $G$ exists; i.e., there is a variety $W$ and a surjective morphism $f^{\prime}: V \rightarrow W$ inducing a bijection between the points of $W$ and the orbits of $G$. Since $Y$, and hence $V$, is irreducible and rational, $W$ is an irreducible rational curve, and by restricting to an open subset of $W$ if necessary we may assume that $W$ is an open subset of a smooth curve $C$ isomorphic to $\mathrm{P}^{1}$.

Thus $f^{\prime}$ induces a morphism $f: V \rightarrow \mathbb{P}^{1}$ which extends to a morphism $\varphi: Z \rightarrow \mathbb{P}^{1}$, where $Z$ is a (possibly trivial) blowing-up of points of $Y$. But $V$ must contain infinitely many $G$-orbits each of whose closures is an irreducible exceptional curve of $Y$. Thus the proper transform $E^{\prime}$ on $Z$ of each such curve $E$ occurs in a distinct fibre of $\varphi$. By Bertini's theorem $[\mathbf{Z 1}, \mathbf{Z 2}]$ it follows that a positive multiple $m\left[E^{\prime}\right]$ of the divisor class $\left[E^{\prime}\right]$ of some such curve $E^{\prime}$ is the divisor class of a fibre of $\varphi$. But this means that $E^{\prime}$ has self-intersection 0 . However, $E^{\prime}$ is a proper transform of $E$ with respect to a birational morphism $Z \rightarrow Y$ and so $\left(E^{\prime}\right) \cdot\left(E^{\prime}\right)<(E) \cdot(E)$ and the latter self-intersection number is -1 . This contradiction concludes the proof.

COROllary (1.4). It never happens that $\operatorname{Aut}^{\#}(X)$ and $\operatorname{Aut}^{*}(X)$ are both infinite.

ProOF. If the latter group were infinite, then $X$ has infinitely many exceptiona. curves, which by the proposition means that Aut ${ }^{\#}(X)$ must be finite.

2. An example with $\operatorname{Aut}^{*}(X)$ infinite. We construct here a complete smooth rational surface $X$ for which $\operatorname{Aut}^{*}(X)$ is infinite but such that $X$ has no antipluricanonical curve. Begin with a nonsupersingular smooth cubic curve $D^{\prime} \subset \mathrm{P}^{2}$. Because $D^{\prime}$ is not supersingular, $\operatorname{Pic}\left(D^{\prime}\right)$ contains a cyclic group $G$ of order 46 . Now identify $D^{\prime}$ with $\operatorname{Pic}^{0}\left(D^{\prime}\right)$ as usual by choosing a flex point of $D^{\prime}$ as the group identity. Let $X^{\prime}$ be the blowing-up of $\mathbb{P}^{2}$ at the points $i p, i=1, \ldots, 8,10$, where $p$ is a point of $D^{\prime}$ which generates $G$ and where $\{i p\}$ are other points of $D^{\prime}$ corresponding to multiples of $p$ in the group $G$.

Denote the proper transform of $D^{\prime}$ on $X^{\prime}$ by $D$. We have the restriction homomorphism $j^{*}: \operatorname{Pic}\left(X^{\prime}\right) \rightarrow \operatorname{Pic}(D)$. It is easy to see that $j^{*}([D])=0$ and therefore $[$ H2, Proposition (1.2)(b), G, Lemma 4] that $D$ moves in a linear system of dimension 1 and without base points. This linear system defines a morphism $\varphi: X^{\prime} \rightarrow P^{1}$, with respect to which $X^{\prime}$ is a minimal elliptic rational surface. By precisely the same argument as is used in example $(2.8)$ of $[\mathbf{H 1}], X^{\prime}$ has no smooth irreducible rational curves of self-intersection -2 . So, from the classification of the fibres of elliptic fibrations $[\mathbf{K}, \mathbf{N r}]$, the fibres of $\varphi$ are irreducible and, since the anticanonical class $[-D]$ is a primitive element of the module $\operatorname{Pic}\left(X^{\prime}\right)$, reduced. Because the Euler characteristic of $X^{\prime}$ is 12, it follows from formula (**) of [D2] that not every fibre of $\varphi$ can be smooth. Thus there is at least one singular fibre (a rational curve with either a simple node or cusp). And by Theorems (3.1) and (4.1)(2) of [H1] it 


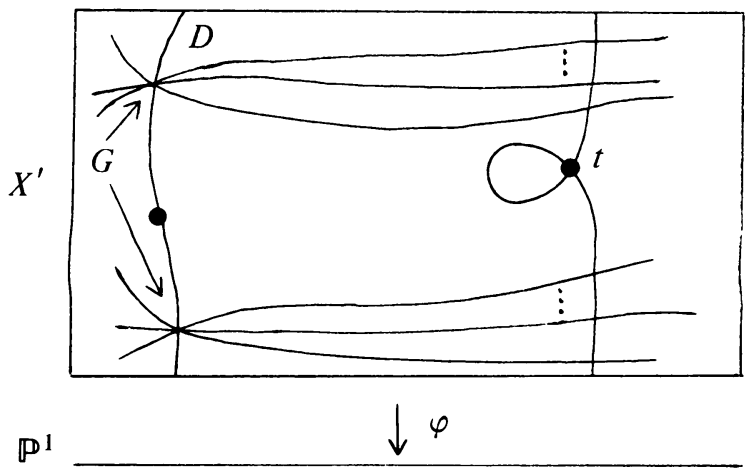

Diagram 2.1

follows, since $G$ is finite, that there is an automorphism $\alpha^{\prime}$ of $X^{\prime}$ that has image in $\operatorname{Aut}^{*}\left(X^{\prime}\right)$ of infinite order. Now $\alpha^{\prime}$ preserves the set of fibres of $\varphi$ since they are anticanonical. But there are only finitely many $\varphi$-fibres $F$ that are singular or for which the order of $j^{*}\left(\operatorname{Pic}\left(X^{\prime}\right)\right) \cap \operatorname{Pic}^{0}(F)$ is less than a given number. (Otherwise, because the sections of $\varphi$ are the irreducible exceptional curves, of which there are infinitely many, there would be two exceptional curves meeting at infinitely many fibres of $\varphi$.) But $D$ is a fibre for which the order of $j^{*}\left(\operatorname{Pic}\left(X^{\prime}\right)\right) \cap \operatorname{Pic}^{0}(D)=G$ is 46 , so by replacing $\alpha^{\prime}$ by a power we can assume that $\alpha^{\prime}$ preserves $D$ and each singular fibre.

Our example $X$ is now obtained by blowing up $X^{\prime}$ at two points: at a singular point $t$ of a singular fibre of $\varphi$; and at a point of $D$ (denote by $X^{\prime \prime}$ the blowing-up of $X^{\prime}$ at the second of these two points). First, $\alpha^{\prime}$ preserves $D$. Now $D$ contains $G$ and $G$ is generated by the points of intersection of $D$ with exceptional curves, so $\alpha^{\prime}$ also preserves $G$. But $G$ is a finite set. Therefore, after replacing $\alpha^{\prime}$ by some power, we may assume that $\alpha^{\prime}$ fixes each element of $G$. Thus the restriction of $\alpha^{\prime}$ to $D$ fixes 46 points of $D$ and hence is trivial. Since each singular fibre has a unique singular point, these points are also fixed by $\alpha^{\prime}$. Thus $\alpha^{\prime}$ lifts to an automorphism $\alpha$ of $X$ and clearly the order of the image of $\alpha$ in $\operatorname{Aut}^{*}(X)$ is infinite.

It only remains to show that $X$ has no antipluricanonical curve. To see this, note that the class $\left[D^{\prime \prime}\right]$ is anticanonical for $X^{\prime \prime}$ and that $-K=\left[D^{\prime \prime}\right]-[F]$, where $K$ is the canonical class for $X,[F]$ is the class of the total transform of $t$ to $X$ and by $\left[D^{\prime \prime}\right]$ we mean here both the class of the proper transform of $D$ to $X^{\prime \prime}$ and its inclusion in $\operatorname{Pic}(X)$. Moreover, $\left[D^{\prime \prime}\right] \cdot\left[D^{\prime \prime}\right]<0$ and $\left[D^{\prime \prime}\right] \cdot[F]=0$. Now suppose for some positive integer $m$ that $-m K$ were effective. Since $\left[D^{\prime \prime}\right] \cdot(-K)=\left[D^{\prime \prime}\right] \cdot\left[D^{\prime \prime}-F\right]<0$ and $D^{\prime \prime}$ is irreducible, we see $-m K-\left[D^{\prime \prime}\right]$ must be effective. By the same reasoning it eventually follows that $-m K-m\left[D^{\prime \prime}\right]=-m[F]$ must be effective, which is absurd since $F$ is effective. Thus $X$ has no antipluricanonical curve.

Diagram 2.1 may give some idea of how this example works. Two fibres of $\varphi$ are displayed vertically. Sections of $\varphi$, which are irreducible exceptional curves, are represented horizontally. There are infinitely many such sections (indeed, the blowings-up of $\{i p: i=1, \ldots, 8,10\}$ give sections which generate a free abelian group of index 3 in the group of sections $[\mathbf{M}]$ ), but they intersect the fibre $D$ in the finitely many points of $G$, as suggested by the diagram. The automorphism $\alpha^{\prime}$ 
fixes each point of $D$ and also fixes the singular point $t$ of the other fibre. For each point of $G, \alpha^{\prime}$ also permutes the sections of $\varphi$ passing through that point. Since $X$ is the surface obtained by blowing up a point of $D$ and the singular point $t$ of the other fibre (represented by the two black dots in the diagram), and since these are $\alpha^{\prime}$-fixed points, $\alpha^{\prime}$ lifts to an automorphism $\alpha$ of $X$.

3. An example with $\operatorname{Aut}^{\#}(X)$ infinite. We now present an example in which $X$ has no antipluricanonical curve but for which nonetheless $\operatorname{Aut}^{\#}(X)$ is infinite. It is similar to the previous example in that we start with a rational surface $X^{\prime}$ having an elliptic fibration (in characteristics 2 or 3 , a quasi-elliptic fibration) and $X$ is obtained by blowing-up a pair of points of $X^{\prime}$ fixed by an infinite subgroup of $\operatorname{Aut}\left(X^{\prime}\right)$. So consider homogeneous coordinates $x, y$, and $z$ on $\mathrm{P}^{2}$. Linear combinations of the cubic forms $z^{3}$ and $x^{2} z+y^{3}$ determine a linear pencil of cubic curves. We have an action of $k^{*}$ on $\mathrm{P}^{2}$ by sending, for each $t \in k^{*}, x, y$, and $z$ to $t^{3} x, t^{2} y$, and $z$, respectively. This action preserves our pencil of cubic curves and fixes the two curves of the pencil given by the forms $z^{3}$ and $x^{2} z+y^{3}$. We take $X^{\prime}$ to be the minimal blowing-up of $P^{2}$ that eliminates the base points of this pencil. The pencil on $X^{\prime}$ is an elliptic (quasi-elliptic in characteristics 2 and 3 ) fibration in which the fibres $F_{1}$ and $F_{2}$ corresponding to the forms $z^{3}$ and $x^{2} z+y^{3}$ have Kodaira types (cf. $[\mathbf{K}, \mathbf{N r}]$ ) $\mathrm{II}^{*}$ and II, respectively. For the generic fibre $C^{*}$ we have the map $j^{*}: \operatorname{Pic}\left(X^{\prime}\right) \rightarrow \operatorname{Pic}\left(C^{*}\right)$ and the group $R$ of rational points of $C^{*}$ is $K^{\perp} / \operatorname{Ker} j^{*}\left[\mathbf{G}\right.$, Lemma 5], where $K^{\perp}$ comprises the divisor classes on $X^{\prime}$ perpendicular to the canonical class $K$. But the components of $F_{1}$ generate both $\operatorname{Ker} j^{*}$ and $K^{\perp}$ so $R$ is trivial. Thus the fibration on $X^{\prime}$ has a single section $E$, the sole irreducible exceptional curve on $X^{\prime}$. Let $p$ and $q$ be the points of $X^{\prime}$ where, respectively, $F_{1}$ and $F_{2}$ meet $E$. Clearly these points are $k^{*}$-invariant. Thus, taking $X$ to be the blowing-up of $X^{\prime}$ at $p$ and $q$, the action of $k^{*}$ lifts to an action on $X$. The fact that $X$ has no antipluricanonical curve follows just as in the previous example, keeping in mind that the fibres of the fibration on $X^{\prime}$ are anticanonical.

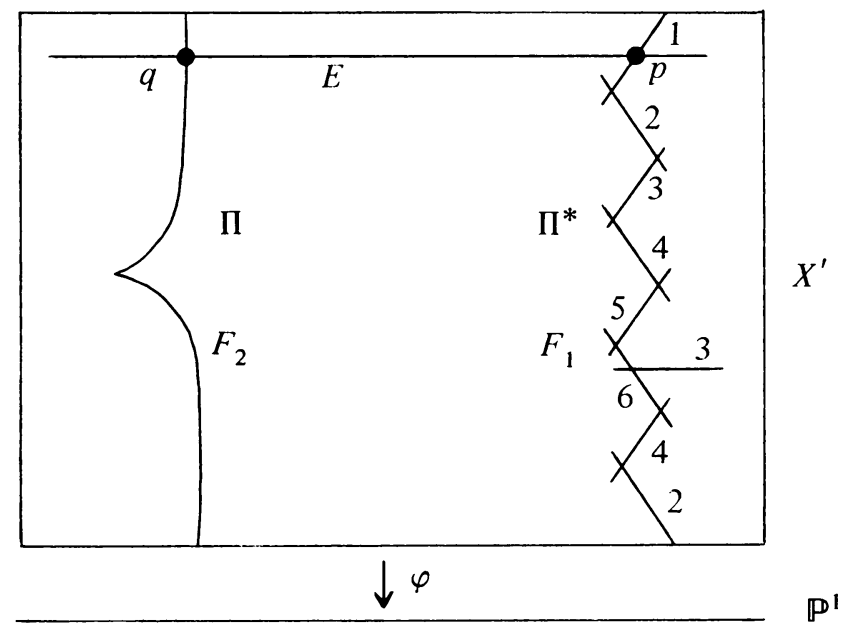

Diagram 3.1 
With the same conventions as before, Diagram 3.1 represents $X^{\prime}$, the large dots being the points to be blown up to obtain $X$. The numbers beside the fibre of type II $^{*}$ indicate the multiplicities of its various components.

\section{REFERENCES}

[D1] I. Dolgachev, On automorphisms of Enriques surfaces, Invent. Math. 76 (1984), 163-177.

[D2] _ The Euler characteristic of a family of algebraic varieties, Math. USSR-Sb. 18 (1972), 303-319.

[G] M. H. Gizatullin, Rational G-surfaces, Math. USSR-Izv. 16 (1981), 103-134.

[H1] B. Harbourne, Blowings-up of $\mathrm{P}^{2}$ and their blowings-down, Duke Math. J. 52 (1985), 124-148.

[H2] _ Complete linear systems on rational surfaces, Trans. Amer. Math. Soc. 289 (1985), 213-226.

[Hu] J. E. Humphreys, Linear algebraic groups, Springer-Verlag, Berlin-Heidelberg-New York, 1975, pp. xiv, 247.

[K] K. Kodaira, On compact analytic surfaces. II, Ann. of Math. 77 (1963), 563-626.

[M] Yu. Manin, The Tate height of points on an Abelian variety, its variants and applications, Izv. Akad. Nauk SSSR Ser. Mat. 28 (1964), 1363-1390. (Russian) (Math. Rev. 30, \#3886)

[N] M. Nagata, On rational surfaces. I, Mem. Coll. Sci. Kyoto (A) 33 (1960), 352-370.

[Nr] A. Néron, Modèles minimaux des variétés abéliennes sur les corps locaux et globaux, Inst. Hautes Études Sci. Publ. Math. 21 (1964).

[R] M. Rosenlicht, A remark on quotient spaces, An. Acad. Brasil Cienĉ. 35 (1963), 486-489.

[Z1] O. Zariski, Introduction to the problem of minimal models in the theory of algebraic surfaces, Publ. Math. Soc. Japan 4 (1958), vii, 89.

[Z2] , Pencils on an algebraic variety and a new proof of a theorem of Bertini, Trans. Amer. Math. Soc. 50 (1941), 48-70. 68588

Department of Mathematics, University of Nebraska, Lincoln, Nebraska 